Ref.: Ms. No. COPLBI-D-19-00047

57: Cell signaling and gene regulation

Current Opinion in Plant Biology

\title{
Is the plant nucleus a mechanical rheostat?
}

Rituparna Goswami ${ }^{\mathrm{a}, \mathrm{b}}$, Atef Asnacios ${ }^{\mathrm{c}}$, Olivier Hamant ${ }^{\mathrm{b} *}$ and Marie-Edith Chabouté $\mathrm{e}^{\mathrm{*}}$

a Institut de biologie moléculaire des plantes, CNRS, Université de Strasbourg, 67084 Strasbourg, France

${ }^{\mathrm{b}}$ Laboratoire de Reproduction et Développement des Plantes, Université de Lyon, UCB Lyon 1, ENS de Lyon, INRA, CNRS, 69364 Lyon, France

c Laboratoire Matières et Systèmes Complexes, Université de Paris, CNRS, Université ParisDiderot, 75013 Paris, France

*Correspondence: marie-edith.chaboute@ibmp-cnrs.unistra.fr ; olivier.hamant@ens-lyon.fr

\begin{abstract}
Beyond its biochemical nature, the nucleus is also a physical object. There is accumulating evidence that its mechanics plays a key role in gene expression, cytoskeleton organization, and more generally in cell and developmental biology. Building on data mainly obtained from the animal literature, we show how nuclear mechanics may orchestrate development and gene expression. In other words, the nucleus may play the additional role of a mechanical rheostat. Although data from plant systems are still scarce, we pinpoint recent advances and highlight some differences with animal systems. Building on this survey, we propose a list of prospects for future research in plant nuclear mechanotransduction and development.
\end{abstract}

\section{Introduction}

Living organisms adapt their physiology and development to environmental cues, notably through transcriptional control. They also respond to internal cues. In particular, growth and shape generate patterns of mechanical stress. In turn, cells resist or yield to mechanical forces by modulating their mechanical properties. This applies to the matrix [1], the cytoskeleton [2], as well as the nucleus [3], with important consequences on gene expression and development [4]. 
Ref.: Ms. No. COPLBI-D-19-00047

57: Cell signaling and gene regulation

Current Opinion in Plant Biology

We recently found that nucleus shape and mechanics depend on osmotic conditions, and that a nuclear regulator controls this response [5]. Here we review key features of nuclear mechanics and mechanotransduction based on data in mammals, yeast, and more recently plants, to highlight shared principles and plant specificities.

\section{The three structural compartments of the nucleus}

The nucleus is surrounded by the nuclear envelope (NE). The NE consists of two lipid bilayer membranes, namely outer (ONM) and inner (INM) nuclear membranes, which are separated by a $\sim 35-50 \mathrm{~nm}$ perinuclear space. These membranes fuse together at the sites of Nuclear Pore Complex (NPC) giving rise to an ultra-donut topology [6]. Both NE components, notably LINC complexes (Linker of Nucleoskeleton and Cytoskeleton) and NPC may contribute to changes of mechanical properties of the nucleus in animals [7]. In plants LINC complexes and NPC have been identified [8].

The nucleoskeleton is connected to the NE by the inner nuclear membrane. It forms a filament meshwork of 10-30 nm composed of coiled-coiled proteins, called lamina in animals and plamina in plants $[9,10]$. While lamins form the lamina in animals, no orthologs of lamins but rather functional homologs have been identified in plants [11-13].

Last, the nucleus contains DNA wrapped around histones to form the nucleosomes. The nucleosomes are organized into 3D higher-order structures, where each chromosome occupies a territory called CT (for chromosome territory) in all eukaryotes, the organization of which can be modulated by mechanical forces [14]. Particularly in Arabidopsis, each CT forms a rosette like structure where transcriptionally active euchromatin loops emanate from the central cluster of condensed inactive heterochromatin which form chromocenters, located at the nuclear periphery $[15,16]$.

Given their direct and indirect roles in transcription, these three compartments have attracted considerable attention in biology. Because they are also physical components, they are the targets of mechanical deformation and regulation, and have become a hot topic of study in biophysics too. 
Ref.: Ms. No. COPLBI-D-19-00047

57: Cell signaling and gene regulation

Current Opinion in Plant Biology

\section{The nucleus as a physical object}

The nucleus has specific mechanical features. When probed through the diffusion of internalized nanoparticles, the nuclear structure reveals transient $\sim 300 \mathrm{~nm}$ sized microdomains with elastic trapping and low elastic moduli ( 20 Pa) and viscosities ( 50 Pa.s) [17]. When the whole nucleus is deformed under constant loading (creep experiment), it displays a time-scale free weak power law behavior, with a viscoelastic modulus ranging from 1 to 10 $\mathrm{kPa}$, and an exponent $\square \sim 0.2-0.3$ [18]. This corresponds to a mechanical behavior dominated by elasticity, with, at a typical time scale of a second, an apparent elastic modulus of about 5 $\mathrm{kPa}$, and an apparent viscosity of $300 \mathrm{~Pa}$.s. More generally, this kind of behavior is observed in many out-of-equilibrium complex systems with multiscale structure and dynamics [19](), like dense suspensions of colloidal particles (muds, shaving foams, emulsions)[20,21].

Importantly, the nucleus viscoelastic modulus correlates with cell identity. For example, during human stem cell differentiation, nuclei stiffen $\sim 6$-fold relative to cell cytoplasm. Consistently, nuclei of primary human fibroblasts are $\sim 2$ times stiffer than nuclei from Marrow-derived human stem cells and their stiffness correlates with the level of expression of lamins [22].

The ability of the nucleus to react to forces also entails energy requirements and dissipation. In that sense, and like most biological objects, the nucleus belongs to the family of active materials [23]. The idea that the nucleus may be an active mechanosensitive element has gained more and more momentum in the past decades [24], also building on the characterization of its structural elements and their physical link to the cytoskeleton [14,25]. In sum, there is now ample evidence that the mechanical properties of the main mechanicallyrelevant elements of the nucleus (nuclear envelope, lamins and chromatin) modulate nuclear mechanosensing, leading to the concept of a nuclear rheostat ([26], Figure 1).

\section{The dynamic mechanical properties of chromatin}

The nucleus displays a plastic behavior and strain hardening (stiffening with increased deformation) reminiscent of the non-linear stress-strain relationship of chromatin fibers [18]. This suggests that chromatin may play a dominating role in nuclear mechanics. In fact, in contrast to the classical view of nuclear mechanics depending mainly on a stiff nucleoskeleton 
Ref.: Ms. No. COPLBI-D-19-00047

57: Cell signaling and gene regulation

Current Opinion in Plant Biology

(lamins) surrounding a softer nucleoplasm (chromatin), recent studies indicate that modifications of histone state are sufficient to dictate nuclear rigidity independently of lamins in animals [27]. Nuclear mechanical properties have also been measured in the plant model Arabidopsis and may be linked to nuclear shape and chromatin status [5].

In mammals, when the balance shifts towards an increase of the transcriptionally less active state, condensed heterochromatin, and decrease of the transcriptional state of the gene-rich active less condensed euchromatin, nuclei become stiffer [27-29]. This response was revealed by playing on chromatin compaction and cytological studies through quantification of relative euchromatin/heterochromatin levels, using either inhibitors of histone post-translational modifications (histone deacetylation, histone methylation, histone demethylation) or genetics (overexpression of nucleosomal binding proteins). In plants, only one study was conducted in differentiated tissues using mutants exhibiting decondensed heterochromatin ( $d d m l$, atxr5atxr6) and no obvious nuclear deformation was observed (nuclear sphericity and volumes). However, the analyses were conducted on fixed nuclei, which can modify the mechanical properties of the nucleus [30]. The nucleus also follows a power-law rheology when subjected to osmosis regulation [18]: isolated nuclei from epidermal cells increase their volume up to $200 \%$ and dilute the DNA content when removing salts in the media, whereas the nucleus becomes wrinkled, due to chromatin condensation, in high salt concentrations.

\section{The dynamic mechanical properties of lamins}

Lamins are nuclear intermediate filaments which can form the fibrous layer in the lamina but they are also found in soluble form in the nucleoplasm [31]. Depending on their locations, lamins have different functions. While lamin $\mathrm{A} / \mathrm{C}$ and $\mathrm{B}$ interact with heterochromatin at the nuclear periphery [32], only lamin A/C interacts with euchromatin via lamin-associated polypeptide $2 \alpha$ allowing a more direct regulation of gene expression [31]. Because lamin A interacts with chromatin, they can regulate nuclear viscosity, whereas lamin B rather modulate nuclear elasticity $[33,34]$. Interestingly using super-resolution microscopy, lamin B1 was shown to form an outer concentric ring, in a curvature-dependent localization to restrain outward protrusions of the stiff lamin A/C network facing the chromatin [35]. Such organization may help to explain the differential mechanical properties of lamins [33,34]. 
Ref.: Ms. No. COPLBI-D-19-00047

57: Cell signaling and gene regulation

Current Opinion in Plant Biology

Both protein levels and folding degree of lamin A seem to scale with tissue stiffness: protein levels are increasing during differentiation in human cells [33] whereas lamins unfolding, through their phosphorylation, was induced in cells growing on soft matrix [36].

In plants, while nucleoskeleton structure was identified by SEM and TEM [37,38] no true lamin exists. Yet specific functional homologs are present [10]. This includes the CRWN (CROWDED NUCLEI) coiled-coil domain proteins found initially in carrot as NMPC (Nuclear Matrix Constituent proteins) [39] and further identified in Arabidopsis as CRWN1-4 [11,40]. While CRWN1 and CRWN4 are exclusively located at the nuclear periphery to mediate chromatin tethering, CRWN2 and CRWN3 are found in the nucleoplasm [40]. Using FRET experiments, CRWN1 was shown to interact with the nucleoplasm N-terminus domain of SUN1 (Sad1-UNC-84, [41]). Other proteins were shown to interact physically with SUN1 using yeast two hybrid, such as the plant specific lamin-like KAKU4 [42] or the chromatin associated protein PWO1 (PROLINE-TRYPTOPHANE-TRYPTOPHANEPROLINE INTERACTOR OF POLYCOMBS1, [43]). In Arabidopsis while CRWN1 and KAKU4 may maintain nuclear morphology through their interaction with the NE $[42,44]$, they can deform the NE independently [42]. Interestingly, genetic interactions between single and double mutants, reveal that $P W O 1$ and $C R W N 1$ control nuclear size and shape in the same genetic pathway and control expression of a similar set of target genes repressed by the repressive histone marks H3K27me3.[43]. In the same trend, LINC complexes were shown to contribute to heterochromatin organisation and transcriptional gene silencing in plants [30]. Yet, very little is known about the contribution of these factors to nuclear mechanics. With the current data, only nuclear shape can be used as a proxy of nuclear mechanical properties. As a future direction of research, the scaling of nuclear deformation and chromatin organization with mechanical stress levels should be investigated in plants. Further studies could also correlate changes in plamina and nuclear mechanics to gene expression, including HI-C data and epigenetic landscape, as well as nuclear translocation of specific transcription factors.

\section{The nuclear envelope as a transducer of cytoplasmic cues}

The NE has its own mechanical properties. The elasticity, viscosity and plasticity of the NE is supported and modulated by several key proteins [45]. The geometric gap between the nuclear membranes is mostly maintained via the SUN1 and SUN2 proteins [46]. SUN1 and SUN2 
Ref.: Ms. No. COPLBI-D-19-00047

57: Cell signaling and gene regulation

Current Opinion in Plant Biology

proteins are located at the INM and interact, via their C-terminus SUN domain, with the KASH (Klarsicht/ANC-1/SYNE homology) domain of KASH family proteins located at the ONM. These SUN-KASH complexes form the LINC complexes and are found across all eukaryotes [46-50]. In the case of vertebrates, SUN1 is also connected to lamins A/C [51].

In a cellular context, the dynamics of actomyosin and microtubules can generate compressive and pulling stresses on the nucleus [52]. The NE plays a key intermediary role. For instance, disrupting the perinuclear actin cap through actin depolymerization leads to an increase of nuclear volume in mouse fibroblasts [53]. Similarly, untethering chromatin from the inner nuclear membrane induces nuclear deformation and reduces nuclear rigidity in S. pombe [54]. In plants, the presence of a large vacuole may further constrain the nucleus in a thin cytoplasmic compartment. Consistently, actin depolymerization in leaf epidermal cells does not change the elongated shape of nuclei [44]. Yet, LINC complexes are associated to actin via Myosin XI-i in plant cells [55] and impaired LINC functions prevent nuclear elongation in differentiated tissues [56,57]. Therefore, the shape of plant nuclei depends on NE factors and the cytoskeleton.

Interestingly, cortical microtubules align with maximal tensile stress directions in plant cell walls $[58,59]$ and cytoplasmic microtubules are connected to the nuclear envelope through the microtubule nucleation complexes and regulatory factors [60,61]. Yet, how cortical stress may affect microtubule nucleation or NE properties is unknown.

\section{The mechanical regulation of gene expression through the nucleus properties}

Because the cytoskeleton and NE components are relatively stiff, cortical mechanical cues can change nucleus shape rapidly, leading to subsequent chromatin changes (Figure 2). For instance, the perinuclear actin cap can induce nuclear deformation mediated by LINC complexes and lamins $\mathrm{A} / \mathrm{C}$ in 30 seconds $[25,62,63]$. Disrupting LINC complexes through SUN1 perturbation in fibroblasts cells leads to different transcriptional responses on soft versus stiff substrates [4]. Unfolding of lamins through their dephosphorylation affect gene expression within 10 minutes [36]. Last, destabilization of lamins under stress induces global chromatin reorganization, release of sequestrated transcription factors as well as 
Ref.: Ms. No. COPLBI-D-19-00047

57: Cell signaling and gene regulation

Current Opinion in Plant Biology

modifications in setting repressive histone marks $\mathrm{H} 3 \mathrm{~K} 27 \mathrm{me} 3$ mediated by the polycomb complex PRC2 [64] (Figure 2).

In parallel to the propagation of stress through the internal structural elements of the cell, cortical cues may indirectly reach the nucleus through protein translocation to the nucleus. In particular, the well-known Hippo pathway-YAP/TAZ involves the traffic of the transcriptional activator B-catenin from the cytoplasm to the nucleoplasm through the NPC [65]. Note that because NPCs are directly connected to nucleoskeleton, chromatin, and LINC complexes [66], the passive nuclear entry of the co-transcriptional factor YAP also depends on the physical deformation of the nucleus [67].

Chromatin is actually a site where mechanical and biochemical cues largely overlap [22,27,29,54]. In response to mechanical stress, changes in gene transcription may be modified either by changes of gene position inside the nucleus or by changes of the chromatin state itself. For instance, direct stretching of chromatin through mechanical forces (using RGD-coated magnetic beads) can induce expression of a GFP-tagged gene in hamster cellular line, and this involves actin and LINC complexes. This is triggered through a change in the spatial chromatin organization, i.e. a change in the location of the transgene [14]. Upon cell mechanical constraint, actomyosin induces the nuclear translocation of HDAC3 leading to decreased level of histone acetylation (permissive mark for gene expression) in mouse embryo fibroblast cells leading to a geometry-dependent transcriptional response. Such response is reversible $[68,69]$. On the contrary, inhibition of actin leads to the recruitment of the histone methyltransferase G9a to the NE and thus increases the level of repressive marks H3K9me2/3 in T-lymphocyte cells [70]. Interestingly, using an active 3D mechano-chemical model, also integrating cell geometric constraints, one can predict nuclear mechanics and architecture patterns [71].

In plants, the situation might be different at the molecular level, knowing for instance that there is no plant homolog of the YAP/TAZ pathway. However, the relationship between nuclear mechanics and gene expression might apply to all kingdoms. In this respect, we recently unraveled a link between nucleus, shape, mechanics and touch-response gene expression in response to hyperosmotic stress [5]. Interestingly, touch-response genes are also induced in response to conditions of changes in light conditions [72]. This is also true when 
Ref.: Ms. No. COPLBI-D-19-00047

57: Cell signaling and gene regulation

Current Opinion in Plant Biology

plants are subjected to sound vibrations [73]. Even though nuclear shape changes have not been explored in these latter cases, one could speculate that the plant nucleus integrate multiple environmental cues in part through its mechanics to regulate gene expression.

\section{Functional implications of nuclear shaping in development}

During development, tissue change their shape or mechanical status, and the resulting mechanical conflicts can transduce mechanical cues to cells and, arguably, nuclei. Although most of the knowledge on nuclear mechanics and mechanotransduction has been generated from studies in single cells in culture, this should also apply to cells in a tissue context. This starts to be addressed [74].

For instance, during epidermal morphogenesis in humans, actin-driven force decreased the level of the lamin-binding protein emerin at the INM inducing a loss of anchoring of heterochromatin at the NE and changes in transcriptional programs mediated by the polycomb PRC2, with important consequences for cell identity [75]. Furthermore, impaired NE functions, notably lamins A, lead to a wide range of diseases called laminopathies, such as muscular dystrophies and progeria [76]. Thus, nuclear mechanics shows functional regulation or correlation through a range of physical and pathological condition.

Plant tissues are usually much stiffer than animal ones, because plant cells are highly pressurized with a turgor pressure in the MPa range, and surrounded by a stiff cell wall. Yet, plant cells are still experiencing mechanical conflicts, the pattern of which is determined by tissue shape and growth. Cortical microtubule arrays play a major role in guiding cellulose synthase complex (CSC) to regulate the cell wall and its physical properties. As mentioned above, cortical microtubules align with the direction of maximal tensile stress in the cell wall, thereby reinforcing the cell wall to resist tensile stress [58]. $\gamma$ tubulins are present at the NE, where they nucleate cytoplasmic microtubules, together with GIP proteins (gamma-tubulin complex protein 3 (GCP3) interacting Proteins) [77]. Interestingly, $\gamma$ tubulins are also found at the INM in a complex with SUN1 and GIPs which form a complex with the centromeric chromatin via CENH3 [78,79]. Although GIPs are conserved across species, they are found on both sides of the NE only in plants. Indeed, GIPs are not only associated with microtubule nucleation complexes outside the nucleus (like their human homolog MOZART1 [80]), but 
Ref.: Ms. No. COPLBI-D-19-00047

57: Cell signaling and gene regulation

Current Opinion in Plant Biology

also with the centromeres close to the inner side of the NE [77,78]. The GIP- $\gamma$ tubulin hub might thus have a specific role in nuclear mechanotransduction in plants. Consistently, the giplgip2 mutant exhibits constitutive induction of touch-response genes [5].

Impaired GIP activity lead to irregular shape and lobulated nuclei in both meristems and differentiated root tissues [77] as well as severe developmental defects, with the presence of callus on differentiated tissues [61]. Such effects are not shared with other NE proteins. In particular, most of the nuclear envelope proteins characterized in LINC complexes identified so far regulate nuclear shape, yet, they do not necessarily induce major developmental defects when impaired [56,57]. This may in part be due to genetic redundancy. For instance, single crwn mutants have no growth defects, whereas crwnlcrwn2 and crwn1crwn2crwn4 exhibit defective development, like stunted plants [40]. When compared to wild type, these mutants exhibit smaller and denser nuclei with fewer, more aggregated, chromocenters. Interestingly, only CRWN1 and CRWN4 allow tethering of heterochromatin at the nuclear periphery [81]. CRWN1 interacts with PWO1, and both control nuclear size [43]. PWO1 physically interacts with the polycomb complex PRC2 mediating H3K27me3 repressive marks [82]. Whereas mechanical regulation of nuclear architecture was linked to cell fate decisions in mammals $[83,84]$, this remains to be explored in plants.

\section{Perspectives for plant science: the role of the nucleus in multi-stress responses}

In contrast to animals, plants exhibit a plastic postembryonic development that largely depends on environmental cues. In line with the established role of nuclear mechanics in animal development, plant development also relies on nuclear mechanotransduction. Yet the exact role of nuclear shape, mechanics and structural elements in signaling and development remains to be investigated. Conducting this research in plants will not only unravel conserved and divergent mechanisms in a walled context, it will also help to understand how the nucleus integrates cues from the environment.

In a recent study in Arabidopsis, we show the importance of nuclear mechanics in roots exposed to osmotic stresses. Throughout mechanical assessment of nucleus stiffness by atomic force microscopy and microrheometry, we revealed that hyperosmotic stress leads to nucleus shrinking, stiffening and chromatin remodeling. We also observed a strong induction 
Ref.: Ms. No. COPLBI-D-19-00047

57: Cell signaling and gene regulation

Current Opinion in Plant Biology

of touch-response genes. Importantly, these responses were reversible upon return to isoosmotic conditions. We propose that chromatin behaves like a mechanosensitive gel in response to osmotic stresses (Figure 3) [5].

Interestingly, the giplgip2 mutant nuclei mimicked a hyper-osmotic phenotype. This further suggests that not only NE envelope proteins play an important role in the plant response to mechanical cues, whether such cues are emanating internally from growth or externally from the environment. Consistently, we found that giplgip2 plants are more resistant to harsh osmotic conditions. This suggests that the absence of GIP may actually prime the plant to hyperosmotic conditions (Figure 3).

Based on these recent results, and building on the comparative analysis of nucleus mechanics and mechanotransduction in animals, we thus propose that the nucleus also acts as a mechanical rheostat in plants. Why would plants be competitive model systems to address this question?

Plant cells do not move. Thus the relation between tissue stress and nucleus behavior is more amenable to be investigated in plants. While turgor pressure and the presence of a stiff plant cell wall represent obvious mechanical differences between plant and animal cells, two important remarks regarding mechanotransduction can be made. First, plant protoplasts, i.e. plant cells without their walls, display mechanical properties that are remarkably similar to that of animal cells. However, these features are dependent on the microtubule cytoskeleton, while they are mainly supported by the actin network in animal cells [2]. Second, in plants, the microtubule network organization and orientation is dependent on both cell shape [85][86] and the direction of maximal stress in the cell wall [58]. Thus, any external or internal cues leading to cell shape changes and/or mechanical stresses in the cell wall, may lead to signal propagation through the microtubule network and the NE to the internal nuclear structure. In this context, the nuclear sensitivity to cell shape [87] and subsequent chromatin modifications $[68,71]$ observed in animal cells could also be at play in plant cells. However, in plant cells, the link between cell shape and nuclear shape and activity might be dependent on microtubules rather than actin, as in animal cells, and the forces needed to induce cell shape modifications and mechanotransduction should be orders of magnitude higher than those involved in animal cell mechanosensing. 
Ref.: Ms. No. COPLBI-D-19-00047

57: Cell signaling and gene regulation

Current Opinion in Plant Biology

Finally, the analysis of multi-stress responses is also facilitated in plants due to their plastic development and sessile nature, meaning that eco-devo study of nuclear mechanotransduction can be envisioned in plants. This may also help to understand plant responses to abiotic and biotic stresses too [88,89]. Altogether, nuclear mechanotrasnduction appears as a new field of research in the plant community, with implications going far beyond a comparison with animal nuclei.

\section{REFERENCES AND RECOMMENDED READING}

Papers of particular interest, published within the period of review, have been highlighted as:

*of special interest

Bi et al., 2017 Genome Res.: Using the nucleoporin NUP136/NUP1 fused to GFP in RE-ChIP experiments in different Arabidopsis tissues, the authors show that 10 to $20 \%$ of chromosome regions are anchored at the nuclear periphery and are enriched in TE and H3K27me3-marked genes.

Nmezi et al., 2019 PNAS: Using high resolution microscopy STORM, the authors show that Lamin B1 forms an outer concentring ring adjacent to the INM, the localization of which is strain-dependent. Lamins B stabilize nuclear shape and prevents protusions from the underlying Lamins A/C network.

Chumova et al., 2019 Cells: In Arabidopsis, the $\gamma$ tubulin initially associated with the microtubule nucleation complexes at the ONM, are found to co-localize with SUN at the INM. They are also present in complexes associated with GIP, suggesting new roles of these proteins at the NE.

Damodaran et al., 2018, Mol Biol Cell: The authors show the importance of fibroblast geometry in changing the chromatin condensation state and the transcriptional response to compressive forces.

Shivashankar, 2019, Curr. Opin. Cell Biol.: In this review, the author discusses the contribution of mechanical cues in changing nuclear architecture and cell fate transitions in mammals. 
Ref.: Ms. No. COPLBI-D-19-00047

57: Cell signaling and gene regulation

Current Opinion in Plant Biology

Miroshnikova et al., 2019, Curr. Opin Gen. Dev: The authors present the latest advances linking mechanics and epigenetic gene regulation during differentiation.

** of outstanding interest

Stephens et al., 2018 Mol Biol. Cell: The authors show that modifications of histone state are sufficient to dictate nuclear rigidity independently of lamin perturbation. For instance, chromatin decompaction via increased acetylation is sufficient to decrease nuclear stiffness without perturbing lamins.

Mikulski et al., 2019 Plant Cell: The authors show that PWO1, which interacts with the polycomb complex PRC2, also interact physically with lamin-like CRWN1. This reveals mechanisms for repressing chromatin at the nuclear periphery in plants.

Hu et al., 2019 Genome biol.: The authors show that lamin-like CRWN1 is needed to anchor repressed chromatin domains to the nuclear periphery in Arabidopsis. CRWN1 has a direct interaction with repressive heterochromatin regions, highlighting chromatin interaction with the plamina in Arabidopsis.

Alisafaei et al., 2019, Proc Natl Acad Sci U S A.: The authors develop a 3D chemomechanical model considering the reciprocity in dynamics between extracellular matrix, cytoskeleton and nucleus using micropatterning experiments.

Goswami et al., 2020 Current Biol. : In this paper, the authors propose that plant nuclei may act as a mechanical rheostat in response to osmotic stress with concomitant changes in nuclear rigidity, shape and mRNA levels of touch-response gene. The absence of GIP may prime the plant to respond to hyperosmotic conditions. 
Ref.: Ms. No. COPLBI-D-19-00047

57: Cell signaling and gene regulation

Current Opinion in Plant Biology

The authors were supported by the Centre National de la Recherche Scientifique (CNRS, defi Mecanobio, NEstress 2016-2018), by Fondation Schlumberger pour l'Education et la Recherche (FSER 2016-2018), by the European Research Council Grants ERC-2013-CoG615739 "MechanoDevo", by the IdEX international PhD program (unista, Strasbourg) and by HFSP Grant 2018, RGP, 009. This study was partially supported by the labex «Who AM I ?», labex ANR-11-LABX- 0071 and the Universite de Paris, Idex ANR-18-IDEX-0001 funded by the French Government through its «investments for the future» program. We apologize to our colleagues whose work we were not able to discuss because of space constraints.

\section{References}

1. Engler AJ, Sen S, Sweeney HL, Discher DE: Matrix elasticity directs stem cell lineage specification. Cell 2006, 126:677-689.

2. Durand-Smet P, Chastrette N, Guiroy A, Richert A, Berne-Dedieu A, Szecsi J, Boudaoud A, Frachisse JM, Bendahmane M, Hamant O, et al.: A comparative mechanical analysis of plant and animal cells reveals convergence across kingdoms. Biophys J 2014, 107:2237-2244.

3. Lovett DB, Shekhar N, Nickerson JA, Roux KJ, Lele TP: Modulation of Nuclear Shape by Substrate Rigidity. Cell Mol Bioeng 2013, 6:230-238.

4. Alam SG, Zhang Q, Prasad N, Li Y, Chamala S, Kuchibhotla R, Kc B, Aggarwal V, Shrestha S, Jones AL, et al.: The mammalian LINC complex regulates genome transcriptional responses to substrate rigidity. Sci Rep 2016, 6:38063.

5. Goswami R, Asnacios A, Milani P, Graindorge S, Houlné G, Mutterer J, Hamant O, Chabouté M-E: Mechanical Shielding in Plant Nuclei. Curr Biol CB 2020, 30:20132025.e3.

6. Torbati M, Lele TP, Agrawal A: Ultradonut topology of the nuclear envelope. Proc Natl Acad Sci 2016, 113:11094-11099.

7. Kirby TJ, Lammerding J: Emerging views of the nucleus as a cellular mechanosensor. Nat Cell Biol 2018, 20:373-381.

8. Fal K, Asnacios A, Chaboute ME, Hamant O: Nuclear envelope: a new frontier in plant mechanosensing? Biophys Rev 2017, doi:10.1007/s12551-017-0302-6.

9. Turgay Y, Eibauer M, Goldman AE, Shimi T, Khayat M, Ben-Harush K, DubrovskyGaupp A, Sapra KT, Goldman RD, Medalia O: The molecular architecture of lamins in somatic cells. Nature 2017, 543:261-264. 
Ref.: Ms. No. COPLBI-D-19-00047

57: Cell signaling and gene regulation

Current Opinion in Plant Biology

10. Ciska M, Moreno Diaz de la Espina S: The intriguing plant nuclear lamina. Front Plant Sci 2014, 5:166.

11. Dittmer TA, Stacey NJ, Sugimoto-Shirasu K, Richards EJ: LITTLE NUCLEI Genes Affecting Nuclear Morphology in Arabidopsis thaliana. Plant Cell 2007, 19:2793-2803.

12. Ciska M, Moreno Diaz de la Espina S: NMCP/LINC proteins: Putative lamin analogs in plants? Plant Signal Behav 2013, 8.

13. Ciska M, Hikida R, Masuda K, De La Espina SMD: Evolutionary history and structure of nuclear matrix constituent proteins, the plant analogues of lamins. J Exp Bot 2019, doi:10.1093/jxb/erz102.

14. Tajik A, Zhang Y, Wei F, Sun J, Jia Q, Zhou W, Singh R, Khanna N, Belmont AS, Wang N: Transcription upregulation via force-induced direct stretching of chromatin. Nat Mater 2016, 15:1287-1296.

15. Fransz P, De Jong JH, Lysak M, Castiglione MR, Schubert I: Interphase chromosomes in Arabidopsis are organized as well defined chromocenters from which euchromatin loops emanate. Proc Natl Acad Sci U A 2002, 99:14584-14589.

16. Bi $\mathrm{X}$, Cheng $\mathrm{YJ}, \mathrm{Hu} \mathrm{B}, \mathrm{Ma} \mathrm{X}, \mathrm{Wu} \mathrm{R}$, Wang JW, Liu C: Nonrandom domain organization of the Arabidopsis genome at the nuclear periphery. Genome Res 2017, 27:1162-1173.

17. Tseng Y, Lee JSH, Kole TP, Jiang I, Wirtz D: Micro-organization and visco-elasticity of the interphase nucleus revealed by particle nanotracking. J Cell Sci 2004, 15:2159-2167.

18. Dahl KN, Engler AJ, Pajerowski JD, Discher DE: Power-law rheology of isolated nuclei with deformation mapping of nuclear substructures. Biophys $J$ 2005, 89:2855-2864.

19. Sollich P, Lequeux F, Hébraud P, Cates ME: Rheology of soft glassy materials. Phys Rev Lett 1997, doi:10.1103/PhysRevLett.78.2020.

20. Höhler R, Cohen-Addad S, Asnacios A: Rheological memory effect in aqueous foam. Europhys Lett 1999, doi:10.1209/epl/11999-00119-4.

21. Purnomo EH, Van Den Ende D, Vanapalli SA, Mugele F: Glass transition and aging in dense suspensions of thermosensitive microgel particles. Phys Rev Lett 2008, doi:10.1103/PhysRevLett.101.238301.

22. Pajerowski JD, Dahl KN, Zhong FL, Sammak PJ, Discher DE: Physical plasticity of the nucleus in stem cell differentiation. Proc Natl Acad Sci U A 2007, 104:15619-15624.

23. Fletcher DA, Geissler PL: Active Biological Materials. Annu Rev Phys Chem 2009, $60:: 469-486$.

24. Wang N, Tytell JD, Ingber DE: Mechanotransduction at a distance: mechanically coupling the extracellular matrix with the nucleus. Nat Rev Mol Cell Biol 2009, 10:7582. 
Ref.: Ms. No. COPLBI-D-19-00047

57: Cell signaling and gene regulation

Current Opinion in Plant Biology

25. Poh YC, Shevtsov SP, Chowdhury F, Wu DC, Na S, Dundr M, Wang N: Dynamic force-induced direct dissociation of protein complexes in a nuclear body in living cells. Nat Commun 2012, 3:866.

26. Thorpe SD, Lee DA: Dynamic regulation of nuclear architecture and mechanics-a rheostatic role for the nucleus in tailoring cellular mechanosensitivity. Nucleus 2017, $8: 287-300$.

27. Stephens AD, Liu PZ, Banigan EJ, Almassalha LM, Backman V, Adam SA, Goldman RD, Marko JF: Chromatin histone modifications and rigidity affect nuclear morphology independent of lamins. Mol Biol Cell 2018, 29:220-233.

28. Furusawa T, Rochman M, Taher L, Dimitriadis EK, Nagashima K, Anderson S, Bustin M: Chromatin decompaction by the nucleosomal binding protein HMGN5 impairs nuclear sturdiness. Nat Commun 2015, 6, 6138.

29. Stephens AD, Banigan EJ, Adam SA, Goldman RD, Marko JF: Chromatin and lamin A determine two different mechanical response regimes of the cell nucleus. Mol Biol Cell 2017, 28:1984-1996.

30. Poulet A, Duc C, Voisin M, Desset S, Tutois S, Vanrobays E, Benoit M, Evans DE, Probst A V, Tatout C: The LINC complex contributes to heterochromatin organisation and transcriptional gene silencing in plants. J Cell Sci 2017, 130:590-601.

31. Gesson K, Rescheneder P, Skoruppa MP, Von Haeseler A, Dechat T, Foisner R: A-type Lamins bind both hetero- and euchromatin, the latter being regulated by laminaassociated polypeptide 2 alpha. Genome Res 2016, 26:462-473.

32. Gruenbaum Y, Foisner R: Lamins: Nuclear Intermediate Filament Proteins with Fundamental Functions in Nuclear Mechanics and Genome Regulation. Annu Rev Biochem 2015, 84:131-164.

33. Swift J, Ivanovska IL, Buxboim A, Harada T, Dingal PC, Pinter J, Pajerowski JD, Spinler KR, Shin JW, Tewari M, et al.: Nuclear lamin-A scales with tissue stiffness and enhances matrix-directed differentiation. Science 2013, 341:1240104.

34. Swift J, Discher DE: The nuclear lamina is mechano-responsive to ECM elasticity in mature tissue. J Cell Sci 2014, 127:3005-3015.

35. Nmezi B, Xu J, Fu R, Armiger TJ, Rodriguez-Bey G, Powell JS, Ma H, Sullivan M, Tu Y, Chen NY, et al.: Concentric organization of A- and B-type lamins predicts their distinct roles in the spatial organization and stability of the nuclear lamina. Proc Natl Acad Sci U S A 2019, 116:4307-4315.

36. Buxboim A, Swift J, Irianto J, Spinler KR, Dingal PC, Athirasala A, Kao YR, Cho S, Harada T, Shin JW, et al.: Matrix elasticity regulates lamin-A,C phosphorylation and turnover with feedback to actomyosin. Curr Biol 2014, 24:1909-1917.

37. Fiserova J, Kiseleva E, Goldberg MW: Nuclear envelope and nuclear pore complex structure and organization in tobacco BY-2 cells. Plant J Cell Mol Biol 2009, 59:243255. 
Ref.: Ms. No. COPLBI-D-19-00047

57: Cell signaling and gene regulation

Current Opinion in Plant Biology

38. Moreno Díaz de la Espina S, Barthellemy I, Cerezuela MA: Isolation and ultrastructural characterization of the residual nuclear matrix in a plant cell system. Chromosoma 1991, doi:10.1007/BF00418244.

39. Masuda K, Xu ZJ, Takahashi S, Ito A, Ono M, Nomura K, Inoue M: Peripheral framework of carrot cell nucleus contains a novel protein predicted to exhibit a long alpha-helical domain. Exp Cell Res 1997, 232:173-181.

40. Wang H, Dittmer TA, Richards EJ: Arabidopsis CROWDED NUCLEI (CRWN) proteins are required for nuclear size control and heterochromatin organization. BMC Plant Biol 2013, 13:200.

41. Graumann K: Evidence for LINC1-SUN associations at the plant nuclear periphery. PLoS One 2014, 9:e93406.

42. Goto C, Tamura K, Fukao Y, Shimada T, Hara-Nishimura I: The Novel Nuclear Envelope Protein KAKU4 Modulates Nuclear Morphology in Arabidopsis. Plant Cell 2014, 26:2143-2155.

43. Mikulski P, Hohenstatt ML, Farrona S, Smaczniak C, Stahl Y, Kalyanikrishna, Kaufmann K, Angenent G, Schubert D: The chromatin-associated protein pwo1 interacts with plant nuclear lamin-like components to regulate nuclear size. Plant Cell 2019, 31:1141-1154.

44. Sakamoto Y, Takagi S: LITTLE NUCLEI 1 and 4 regulate nuclear morphology in Arabidopsis thaliana. Plant Cell Physiol 2013, 54:622-633.

45. Rowat AC, Foster LJ, Nielsen MM, Weiss M, Ipsen JH: Characterization of the elastic properties of the nuclear envelope. $J$ R Soc Interface 2005, 2:63-69.

46. Crisp M, Liu Q, Roux K, Rattner JB, Shanahan C, Burke B, Stahl PD, Hodzic D: Coupling of the nucleus and cytoplasm: role of the LINC complex. J Cell Biol 2006, 172:41-53.

47. Graumann K, Runions J, Evans DE: Characterization of SUN-domain proteins at the higher plant nuclear envelope. Plant J 2010, 61:134-144.

48. Zhou X, Graumann K, Evans DE, Meier I: Novel plant SUN-KASH bridges are involved in RanGAP anchoring and nuclear shape determination. J Cell Biol 2012, 196:203-211.

49. Zhou X, Graumann K, Wirthmueller L, Jones JDG, Meier I: Identification of unique SUN-interacting nuclear envelope proteins with diverse functions in plants. J Cell Biol 2014, 205:677-692.

50. Murphy SP, Simmons CR, Bass HW: Structure and expression of the maize (Zea mays L.) SUN-domain protein gene family: evidence for the existence of two divergent classes of SUN proteins in plants. BMC Plant Biol 2010, 10:269.

51. Haque F, Lloyd DJ, Smallwood DT, Dent CL, Shanahan CM, Fry AM, Trembath RC, Shackleton S: SUN1 interacts with nuclear lamin A and cytoplasmic nesprins to provide 
Ref.: Ms. No. COPLBI-D-19-00047

57: Cell signaling and gene regulation

Current Opinion in Plant Biology

a physical connection between the nuclear lamina and the cytoskeleton. Mol Cell Biol 2006, 26:3738-3751.

52. Gundersen GG, Worman HJ: Nuclear positioning. Cell 2013, 152:1376-1389.

53. Kim DH, Li B, Si F, Phillip JM, Wirtz D, Sun SX: Volume regulation and shape bifurcation in the cell nucleus. J Cell Sci 2015, 129:457.

54. Schreiner SM, Koo PK, Zhao Y, Mochrie SGJ, King MC: The tethering of chromatin to the nuclear envelope supports nuclear mechanics. Nat Commun 2015, 6:7159.

55. Tamura K, Iwabuchi K, Fukao Y, Kondo M, Okamoto K, Ueda H, Nishimura M, HaraNishimura I: Myosin XI-i links the nuclear membrane to the cytoskeleton to control nuclear movement and shape in Arabidopsis. Curr Biol 2013, 23:1776-1781.

56. Tamura K, Goto C, Hara-Nishimura I: Recent advances in understanding plant nuclear envelope proteins involved in nuclear morphology. $J$ Exp Bot 2015, 66:1641-1647.

57. Meier I, Richards EJ, Evans DE: Cell Biology of the Plant Nucleus. TL - 68. Annu Rev Plant Biol 2017, 68 VN-r:139-172.

58. Hamant O, Heisler MG, Jonsson H, Krupinski P, Uyttewaal M, Bokov P, Corson F, Sahlin P, Boudaoud A, Meyerowitz EM, et al.: Developmental patterning by mechanical signals in Arabidopsis. Science 2008, 322:1650-1655.

59. Hamant $\mathrm{O}$, Inoue $\mathrm{D}$, Bouchez $\mathrm{D}$, Dumais J, Mjolsness E: Are microtubules tension sensors? Nat Commun 2019, doi:10.1038/s41467-019-10207-y.

60. Janski N, Herzog E, Schmit AC: Identification of a novel small Arabidopsis protein interacting with gamma-tubulin complex protein 3. Cell Biol Int 2008, 32:546-548.

61. Janski N, Masoud K, Batzenschlager M, Herzog E, Evrard JL, Houlne G, Bourge M, Chaboute ME, Schmit AC: The GCP3-interacting proteins GIP1 and GIP2 are required for gamma-tubulin complex protein localization, spindle integrity, and chromosomal stability. Plant Cell 2012, 24:1171-1187.

62. Chambliss AB, Khatau SB, Erdenberger N, Robinson KD, Hodzic D, Longmore GD, Wirtz D, Robinson DK, Hodzic D, Longmore GD, et al.: The LINC-anchored actin cap connects the extracellular milieu to the nucleus for ultrafast mechanotransduction. Sci Rep 2013, 3:1087.

63. Lombardi ML, Jaalouk DE, Shanahan CM, Burke B, Roux KJ, Lammerding J: The interaction between nesprins and sun proteins at the nuclear envelope is critical for force transmission between the nucleus and cytoskeleton. J Biol Chem 2011, 286:2674326753.

64. Osmanagic-Myers S, Foisner R: The structural and gene expression hypotheses in laminopathic diseases - Not so different after all. Mol Biol Cell 2019, 30:1786-1790. 
Ref.: Ms. No. COPLBI-D-19-00047

57: Cell signaling and gene regulation

Current Opinion in Plant Biology

65. Dupont S, Morsut L, Aragona M, Enzo E, Giulitti S, Cordenonsi M, Zanconato F, Le Digabel J, Forcato M, Bicciato S, et al.: Role of YAP/TAZ in mechanotransduction. Nature 2011, 474:179-183.

66. Jahed Z, Soheilypour M, Peyro M, Mofrad MR: The LINC and NPC relationship - it's complicated! TL - 129. J Cell Sci 2016, 129 VN-:3219-3229.

67. Elosegui-Artola A, Andreu I, Beedle AEM, Lezamiz A, Uroz M, Kosmalska AJ, Oria R, Kechagia JZ, Rico-Lastres P, Le Roux AL, et al.: Force Triggers YAP Nuclear Entry by Regulating Transport across Nuclear Pores. Cell 2017, 171:1397-1410 e14.

68. Jain N, Iyer K V, Kumar A, Shivashankar G V: Cell geometric constraints induce modular gene-expression patterns via redistribution of HDAC3 regulated by actomyosin contractility. Proc Natl Acad Sci U A 2013, 110:11349-11354.

69. Damodaran K, Venkatachalapathy S, Alisafaei F, Radhakrishnan A V., Jokhun DS, Shenoy VB, Shivashankar G V.: Compressive force induces reversible chromatin condensation and cell geometry-dependent transcriptional response. Mol Biol Cell 2018, 29:3039-3051.

70. Zhang X, Cook PC, Zindy E, Williams CJ, Jowitt TA, Streuli CH, MacDonald AS, Redondo-Muñoz J: Integrin $\alpha 4 \beta 1$ controls G9a activity that regulates epigenetic changes and nuclear properties required for lymphocyte migration. Nucleic Acids Res 2015, 44:3031-3044.

71. Alisafaei F, Jokhun DS, Shivashankar G V, Shenoy VB: Regulation of nuclear architecture, mechanics, and nucleocytoplasmic shuttling of epigenetic factors by cell geometric constraints. Proc Natl Acad Sci U A 2019, 16:13200-13209.

72. Lee D, Polisensky DH, Braam J: Genome-wide identification of touch- and darknessregulated Arabidopsis genes: a focus on calmodulin-like and XTH genes. New Phytol 2005, 165:429-444.

73. Ghosh R, Mishra RC, Choi B, Kwon YS, Bae DW, Park S-CC, Jeong M-JJ, Bae H: Exposure to Sound Vibrations Lead to Transcriptomic, Proteomic and Hormonal Changes in Arabidopsis. Sci Rep 2016, 6:33370.

74. Fernandez-Sanchez M-E, Brunet T, Röper J-C, Farge E: Mechanotransduction's Impact on Animal Development, Evolution, and Tumorigenesis. Annu Rev Cell Dev Biol 2015, 31:373-397.

75. Le HQ, Ghatak S, Yeung C-YCY, Tellkamp F, Günschmann C, Dieterich C, Yeroslaviz A, Habermann B, Pombo A, Niessen CM, et al.: Mechanical regulation of transcription controls Polycomb-mediated gene silencing during lineage commitment. Nat Cell Biol 2016, 18:864-875.

76. Horn HF, Brownstein Z, Lenz DR, Shivatzki S, Dror AA, Dagan-Rosenfeld O, Friedman LM, Roux KJ, Kozlov S, Jeang K-T, et al.: The LINC complex is essential for hearing. $J$ Clin Invest 2013, 123:740-750. 
Ref.: Ms. No. COPLBI-D-19-00047

57: Cell signaling and gene regulation

Current Opinion in Plant Biology

77. Batzenschlager M, Masoud K, Janski N, Houlne G, Herzog E, Evrard JL, Baumberger N, Erhardt M, Nomine Y, Kieffer B, et al.: The GIP gamma-tubulin complex-associated proteins are involved in nuclear architecture in Arabidopsis thaliana. Front Plant Sci 2013, 4:480.

78. Batzenschlager M, Lermontova I, Schubert V, Fuchs J, Berr A, Koini MA, Houlne G, Herzog E, Rutten T, Alioua A, et al.: Arabidopsis MZT1 homologs GIP1 and GIP2 are essential for centromere architecture. Proc Natl Acad Sci U A 2015, 112:8656-8660.

79. Chumová J, Kourová H, Trögelová L, Halada P, Binarová P: Microtubular and Nuclear Functions of $\gamma$-Tubulin: Are They LINCed? Cells 2019, 8:259.

80. Hutchins JRA, Toyoda Y, Hegemann B, Poser I, Heriche JK, Sykora MM, Augsburg M, Hudecz O, Buschhorn BA, Bulkescher J, et al.: Systematic analysis of human protein complexes identifies chromosome segregation proteins. Science 2010, 328:593-599.

81. Hu B, Wang N, Bi X, Karaaslan ES, Weber AL, Zhu W, Berendzen KW, Liu C: Plant lamin-like proteins mediate chromatin tethering at the nuclear periphery. Genome Biol 2019, 20:1-18.

82. Hohenstatt ML, Mikulski P, Komarynets O, Klose C, Kycia I, Jeltsch A, Farrona S, Schubert D: PWWP-DOMAIN INTERACTOR OF POLYCOMBS1 interacts with polycomb-group proteins and histones and regulates arabidopsis flowering and development. Plant Cell 2018, 30:117-133.

83. Shivashankar G V: Mechanical regulation of genome architecture and cell-fate decisions. Curr Opin Cell Biol 2019, 56:115-121.

84. Miroshnikova YA, Cohen I, Ezhkova E, Wickström SA: Epigenetic gene regulation, chromatin structure, and force-induced chromatin remodelling in epidermal development and homeostasis. Curr Opin Genet Dev 2019, 55:46-51.

85. Ambrose C, Allard JF, Cytrynbaum EN, Wasteneys GO: A CLASP-modulated cell edge barrier mechanism drives cell-wide cortical microtubule organization in Arabidopsis. Nat Commun 2011, 2:430.

86. Durand-Smet P, Spelman TA, Meyerowitz EM, Jönsson H: Cytoskeletal organization in isolated plant cells under geometry control. Proc Natl Acad Sci U S A 2020, doi:10.1073/pnas.2003184117.

87. Versaevel M, Grevesse T, Gabriele S: Spatial coordination between cell and nuclear shape within micropatterned endothelial cells. Nat Commun 2012, doi:10.1038/ncomms 1668 .

88. Moulia B, Coutand C, Julien J-LL: Mechanosensitive control of plant growth: bearing the load, sensing, transducing, and responding. Front Plant Sci 2015, 6:52.

89. Coutand C: The effect of mechanical stress on plant susceptibility to pests: A mini opinion review. Plants 2020, doi:10.3390/plants9050632. 
Ref.: Ms. No. COPLBI-D-19-00047

57: Cell signaling and gene regulation

Current Opinion in Plant Biology

\section{Figure legends}

Figure 1: Changes of cell and nuclear shapes under mechanical stress.

Under high cell stress, the nucleus is more compact with nucleoskeleton enrichment and spatial chromatin reorganization

\section{Figure 2: The features of chromatin under mechanical stress}

Changes of nuclear architecture under mechanical stress lead to chromatin condensation, clustering of chromocenters, repressive histone mark modifications inducing altered accessibility for the transcription of differentiated gene, and release of transcription factors from the nuclear periphery

\section{Figure 3: Nuclear mechanical response in plants under hyperosmotic stress}

Upon hyperosmotic stress, the nucleus is more compact with clustered chromocenters and induction of touch-response gene expression. The nuclear envelope proteins GIPs may act as negative regulators of this response. 
Low stress
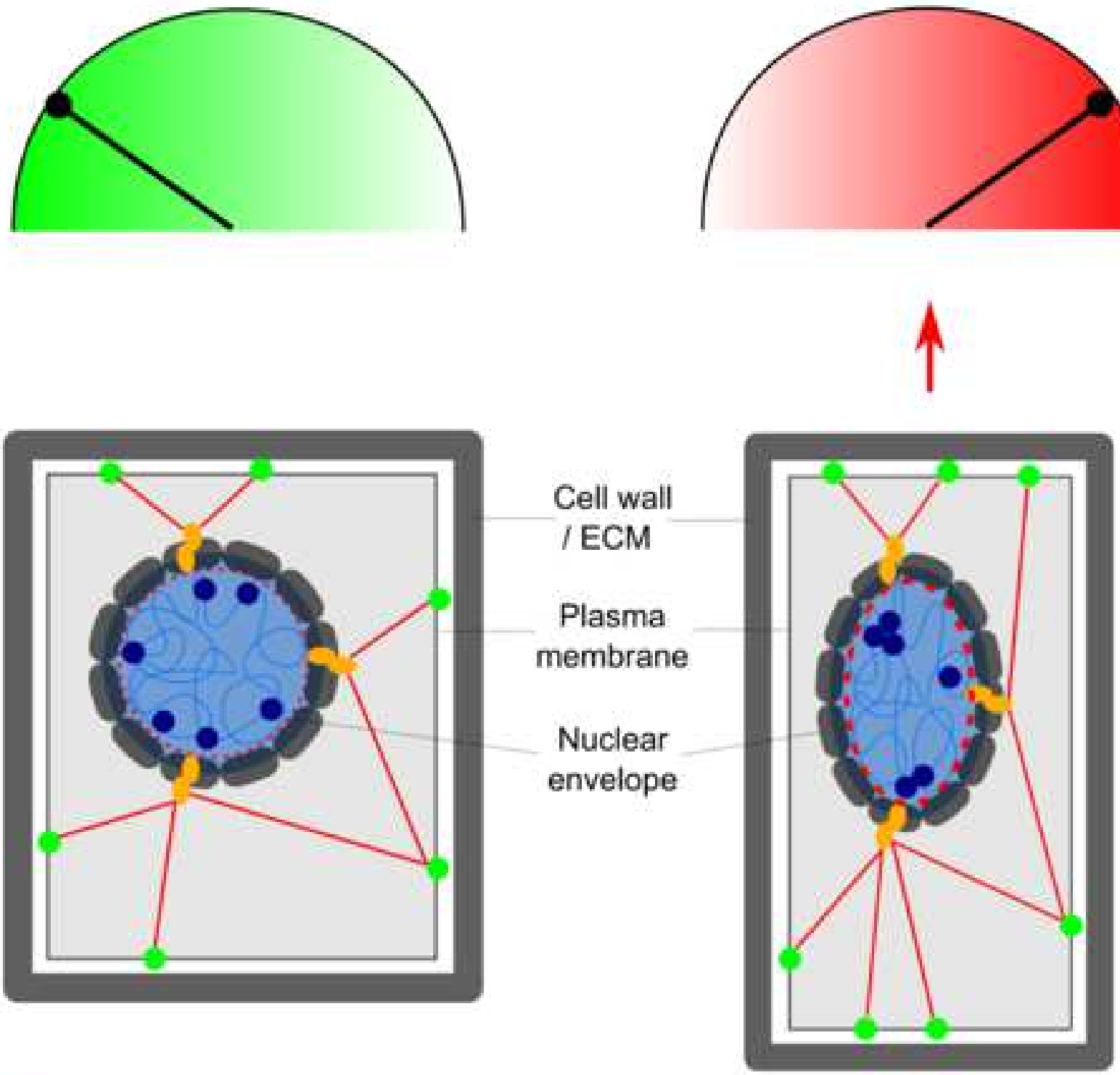

High stress

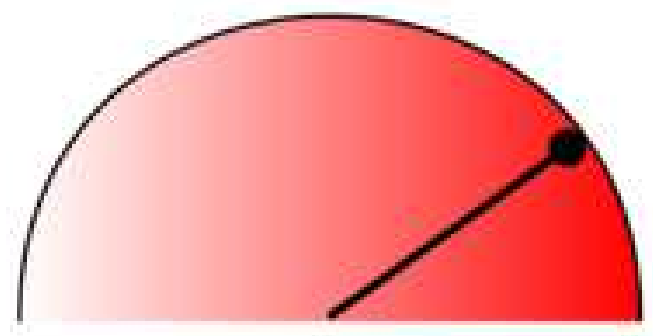

$\uparrow$

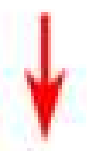

Cortical mechanosensor LINC complex

- Chromocenter

- Cytoskeleton (actin filament/microtubule)

.... Nucleoskeleton (lamina/plamina) 

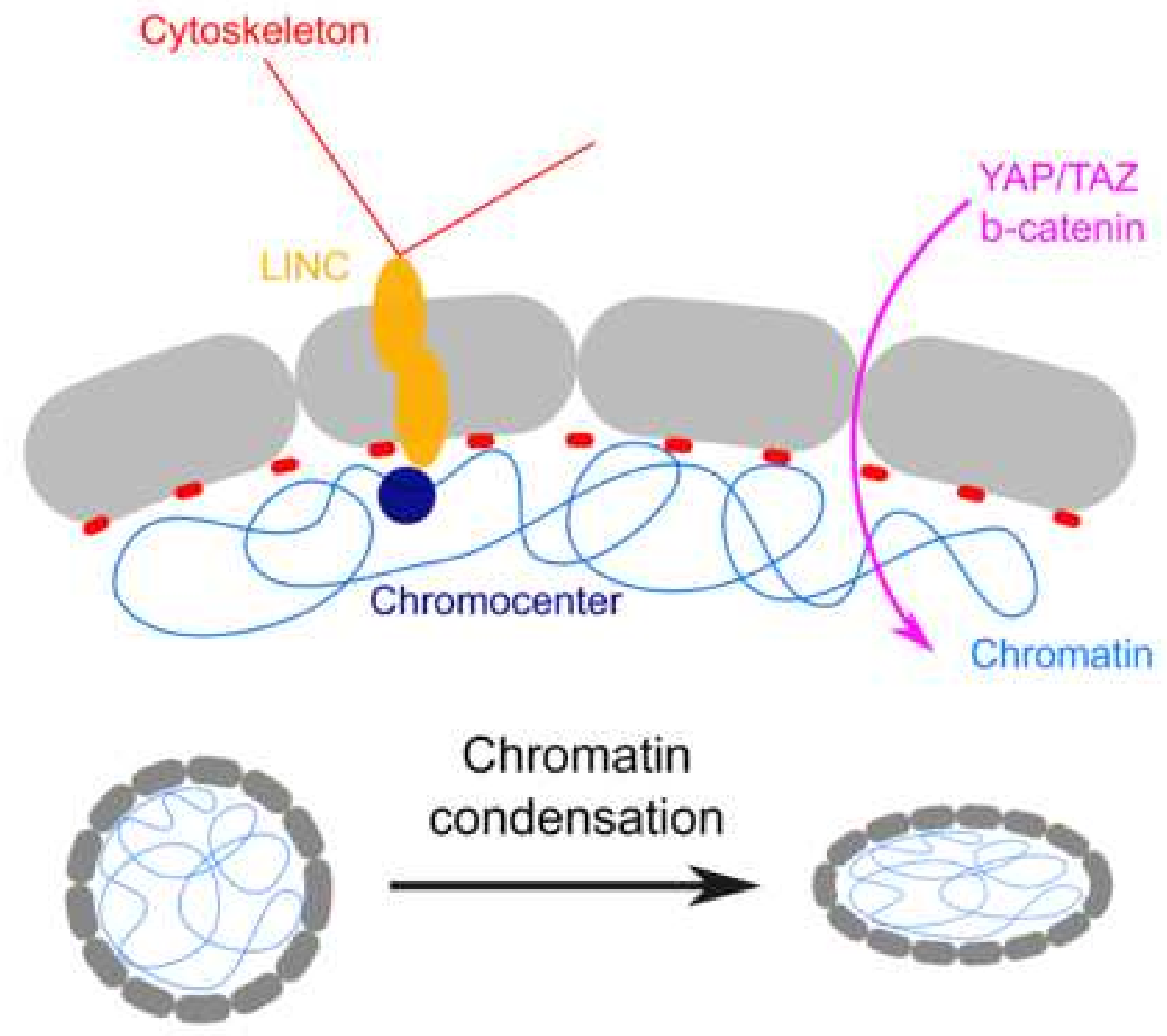

Chromatin
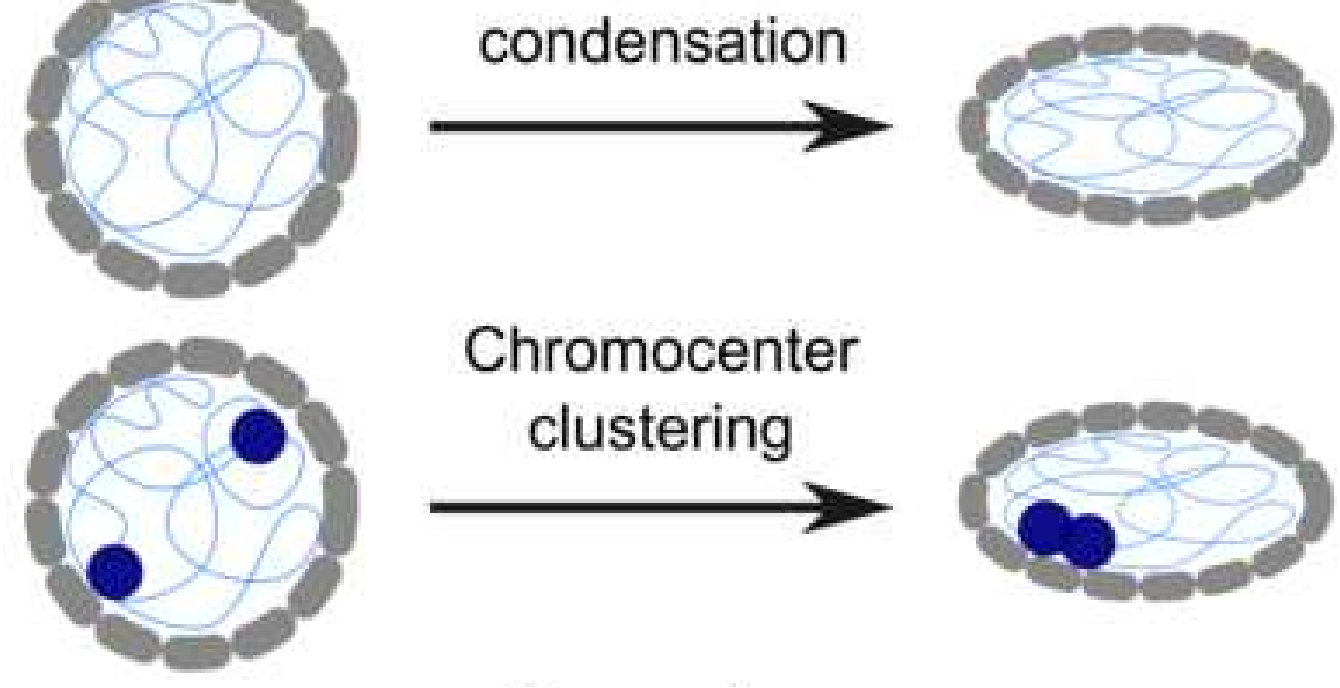

Chromocenter clustering

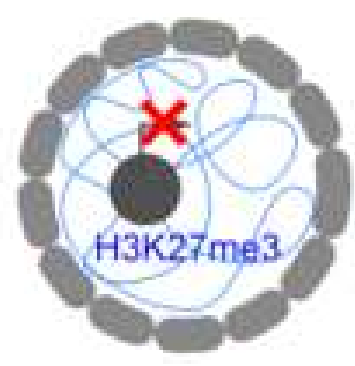

\section{Repressive} histone marks

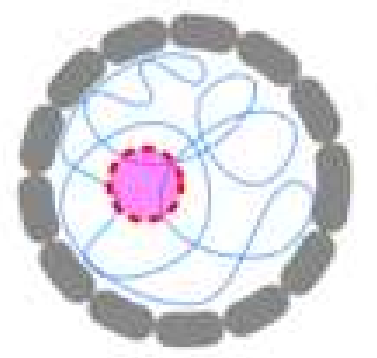

Release of
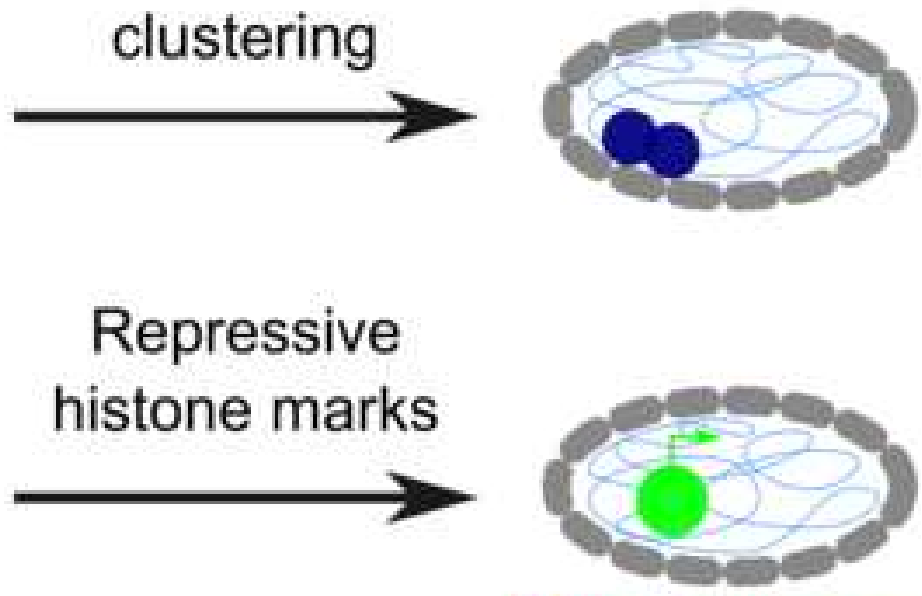

Differentiation gene transcription factors
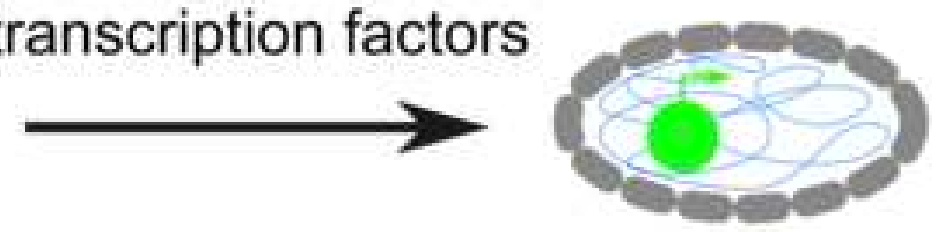

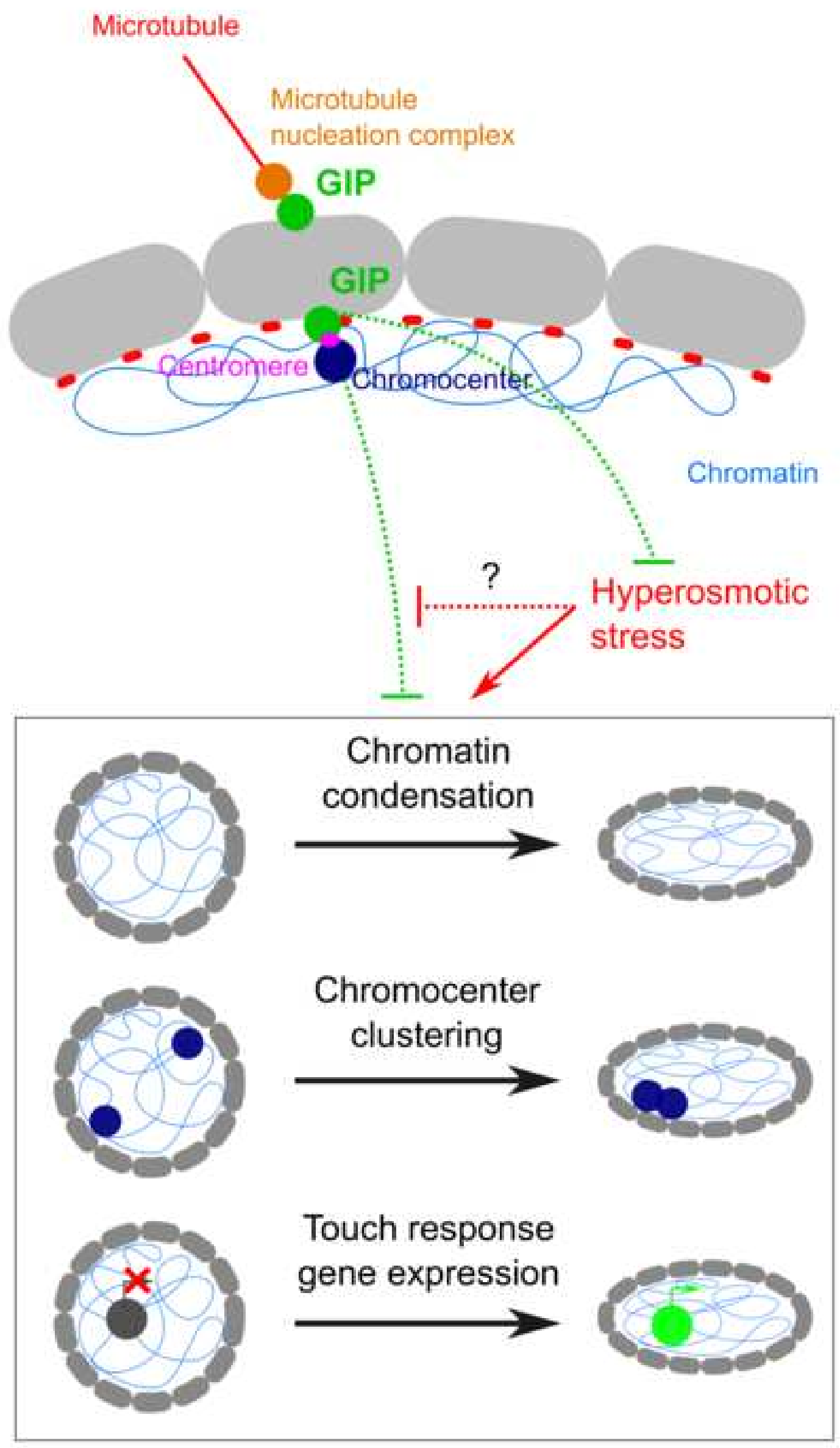